\title{
Analysis of Concordance Between Parent Proxy and Child Self-report of KIDSCREEN-10 Health-related Quality of Life Questionnaire in an Elementary School-based Wellness Program: A Pilot Focus Group Study
}

\author{
Bongsam Choi, MPH, PhD, PT \\ Department of Physical Therapy, College of Health and Welfare, Woosong University, Daejeon, Korea
}

\author{
Article Info \\ Received March 30, 2021 \\ Revised April 20, 2021 \\ Accepted April 20, 2021 \\ Corresponding Author \\ Bongsam Choi \\ E-mail: bchoi@wsu.ac.kr \\ https://orcid.org/0000-0002-0165-4941
}

\section{Key Words}

Proxy

Quality of life

Self report

Treatment outcome
Background: Researchers have previously commenced examining the degree of concordance between parent proxy and child self-reports on health-related quality of life (HRQOL) of many disease and impairment populations.

Objects: To explore the differences between parent proxy and child self-reports on the HRQOL using Korean version of KIDSCREEN-10 questionnaires for applying to elementary school children and their parents who participated for a school-based wellness program.

Methods: The focus groups were recruited for a school-based wellness program by implementing the following wellness services: 1) referring to a screening session for detecting potential posture-related musculoskeletal problems and 2) recommending home exercise programs. Before a primary field testing for the program, two focus groups were formed with a group of 9 parents and their 9 elementary school children aged 8-10 years of age. The parent proxy and child self-report versions of KIDSCREEN-10 questionnaires were administered to both groups after completion of the wellness program. Item level Rasch rating scale analysis was applied to compute logit scales of KIDSCREEN-10 questionnaire. Intraclass correlation coefficients (ICCS) and scatterplot of item difficulty between two reports were analyzed.

Results: For fit statistics of parent proxy report, all items except 4 items (i.e., psychological well-being, mood/emotions, self-perception, parent relation) were found to be acceptable. For fit statistics of child self-report, all items except 3 items (i.e., psychological well-being, autonomy and home life) were acceptable. The relationship between two reports using ICCS were ranging from weak to very strong at $p=0.05$ (i.e, ICCs $=0.011$ to 0.905 ). Scatterplot analysis between two reports showed a major disparity on self-perception item at $95 \%$ confidence intervals.

Conclusion: Both item level analyses and ICC comparisons provided a disparity between parent proxy and child self-reports of the HRQOL on self-perception item after competing a school-based wellness program. Therapist should consider the item as part of the HRQOL assessment.

\section{INTRODUCTION}

School-based wellness programs for children's poor posture are generally designed to enhance educational attainment through improved school health via parent and teacher. Many efforts have been made in trying to track children's school health by providing health campaigns such as wellness programs for detecting musculoskeletal imbalances [1-3]. While it is easy to address why those wellness programs should be implemented in early childhood, the impact of those programs on child's health-related quality of life (HRQOL) has been an issue due to the level of agreement between the perspectives of parents and their children. Although parent proxy and child self-reports may both contribute important information on child's HRQOL, parent reports may improve the feasibility of monitoring the status of HRQOL when their children are not available to provide optimal reports [4]. Parent proxy report is deemed to be the most useful resource as parents are the most familiar with their child's health and life [5]. Research on child's view on HRQOL used to rely on parent or other proxy 
perceptions. However, children's view on their health status is now appreciated and should be considered rather than being inferred from proxy reports [6].

Many factors can cause the disparity between parent and child's view about the HRQOL concepts due to inherent limitations such as different interpretations of HRQOL items. Score disparity between parent proxy and child self-reports have been reported as a major challenge in measuring child's HRQOL [7]. While there have been acceptable levels of agreement for domains reflecting physical functions/symptoms, poor agreements have also noted for domains reflecting more social or emotional concepts [8-10]. Due to children's cognitive immaturity, in general, limited social experiences or dependency to parent, parent proxy reports are considered to be more able to reflect their children's HRQOL [11]. However, in an attempt to capture two different views on the HRQOL, both parent and child self-reports have increasingly been used when developing HRQOL assessments.

Of nearly 30 questionnaires for measuring children's HRQOL are now available for use, the KIDSCREEN-10 questionnaire is a set of mostly accepted questionnaires receiving the most attention [4]. The questionnaires have been translated into at least 38 languages and used in more than 50 well-being studies in children aged from 8-18 years. There are three various versions of KIDSCREEN-10 questionnaire with 52, 27 and 10 items available and 5 point-point Likert type responses to assess the frequency of the concept of HRQOL related dimensions. The KIDSCREEN-10-10 questionnaire covers the concepts of physical and psychological well-being, moods/emotions, selfperception, autonomy, home life, parent relation, peers/social support, school environment and concentration/learning dimensions. In addition, the KIDSCREEN-10 questionnaires are available in parent proxy and child self-report versions providing a global HRQOL status.

Most classical test theory (CTT)-based questionnaires, in general, can cause measurement problems such as ceiling or floor effects [12]. For example, if the items of KIDSCREEN-10 questionnaire are too easy to be viewed by parents or children, outcome scores cluster toward the high end (i.e., the ceiling effects). The opposite, when the items are too difficult to be challenged for parents or children, is the floor effects. These psychometric deficits can be arisen from the measurement problem where unmatched distinction between item difficulties of questionnaire and respondents (i.e., parents and chil- dren) occurs. For example, when challenging items of the KIDSCREEN-10 are administered to respondents with low status of HRQOL or vice versa, this results in the measurement errors [13]. However, most HRQOL questionnaires, as well as the KIDSCREEN-10 questionnaire, do not have adequate breadth for the HRQOL construct with fixed number of items [12-14]. If such measurement problem exist, one may consider to have a large number of items within the questionnaire. However, it is not desirable to have many items in a questionnaire with respect to measurement efficiency [13]. To overcome these limitations of existing CTT-based questionnaires, authors encouraged using Rasch and item response theory (IRT) approaches which focus on the psychometric properties of individual items of a HRQOL questionnaire instead of the questionnaire as a whole [13,14]. The IRT methodology has increasingly been used to test the psychometric properties of the KIDSCREEN-10 questionnaire [15]. Analysis on individual items using Rasch modelling can estimate the probability that respondents will select a particular rating of the individual item. The item difficulty produced by the probability can serve as a means of validating the dimensional structure of a questionnaire. Thus, with an IRT methodology focusing on individual items, it is necessary to investigate whether the disparity between parent proxy and child self-report can be resolved, and to determine what methodologies are best for pursuing the purposes. Further, the small study results can at least be clued to the disparity with the present pilot trial.

The purposes of this study are to investigate; 1) hierarchical item difficulty and fit statistic analysis, 2) concordance and 3) scatterplots between parent proxy and child self-report versions of the KIDSCREEN-10 questionnaire at the time of completion of an elementary school-based wellness program.

\section{MATERIALS AND METHODS}

\section{Instrumentation}

Ten items of the KIDSCREEN-10 used for the present study were selected from the Korean version of KIDSCREEN-52 validated by Hong et al. [16]. The 10 items are rated on a fivepoint rating scale for the frequency of HRQOL related experiences: 1) never, 2) seldom, 3) sometimes, 4) often and 5) always. Two versions of KIDSCREEN-10 questionnaires, parent proxy and child self-reports, were administered to two parent and child focus groups after completion of the school-based 
wellness program. The KIDSCREEN-10 questionnaire consisted of 10 dimensions that are designed to measure general status relevant to HRQOL experiences from the program.

With either the frequency of behavior/feelings or intensity of an attitude, the original version of the KIDSCREEN-10 questionnaire was developed with 52 items with 10 dimensions. The number of items was reduced by 27 with 5 dimensions. Later, the KIDSCREEN-10-10 was created to assess the general status of HRQOL with 10 items designating 10 relevant dimensions (Table 1). Since items of moods/emotions (item 3) and self-perception (item 4) were negatively phrased, those items were reversely scored for statistical analysis in the study. Thus, higher score of the KIDSCREEN-10 questionnaire indicates higher status of HRQOL. The parent proxy and child selfreport versions were identically phrased except subject in sentence. That is, all sentences were phrased like "have your child felt fit and well?" for parent proxy version versus "have you felt fit and well?" for child self-report version.

\section{Focus Groups Selection}

A total of 22 subjects for two focus groups, 11 parents and 11 children, were recruited from October 30, 2019 to November 20, 2019 at a local elementary school, Daejeon, Republic of Korea. All subjects who wanted to participated for schoolbased wellness program at the elementary school were introduced with detail information of the study including informed consent and the conflict of interests notifying potential subjects of no benefits and penalties regardless of participating in the study. The wellness program was designed to promote educational performances by which the children can learn how to properly maintain their posture, avoid potential injuries and learn self-exercise programs with parent. The program includes screening services for any potential postural imbalances and detail health examinations for recommending ideal posture, followed by implementing home exercise program if necessary. Scores from two children and parents, two for each group, were excluded from all statistical analyses due to incomplete data sets. Of the 18 subjects completed the KIDSCREEN-10, less than 33.3 percent were female $(n=3)$ and more than 66.7 percent were male $(n=6)$ with an average age of 9 children ranging from 8 through 10 years of age. All subjects of parent group were children's mothers with an average age of 37. No one were reported any cognitive deficits to report their children's HRQOL. None of the children had reported any previous injuries or diseases. The study was approved by the Institutional Review Board of College of Health and Welfare, Woosong University (approval No. 1041549-190409-SB-73).

\section{Data Analysis}

Scores of the KIDSCREEN-10 questionnaires were analyzed with Winsteps software program version 3.57.2 (Winsteps.com, Chicago, IL, USA) using rating scale model for: 1) item difficulty calibrations in logit scales, 2) fit statistics for dimensionality and 3) scatterplots between parent proxy versus child self-reports. The goodness of fit statistics for the Rasch rating scale model, 1-parameter item response theory model, were examined for how well a particular item fit to the Rasch model. The criteria for fit statistics were determined by Fox and Bond's suggestion, where mean squares are $\geq 1.4$ and $\leq 0.6$ [17]. If fit statistics of items were either too high or low, the item was considered to

Table 1. Item interpretation of KIDSCREEN-10 questionnaire

\begin{tabular}{|c|c|}
\hline Items & Interpretation \\
\hline Item 1 Physical well-being & The level of the child's physical activity, energy, and fitness such as ability to get around the home and school \\
\hline Item 2 Psychological well-being & $\begin{array}{l}\text { The level of the child's positive emotions and satisfaction with life experienced by the individual such as happiness, } \\
\text { joy, and cheerfulness }\end{array}$ \\
\hline Item 3 Mood/emotions & $\begin{array}{l}\text { How much the child experiences depressive moods/emotions and stressful feelings such as loneliness, sadness, } \\
\text { sufficiency/insufficiency, and resignation }\end{array}$ \\
\hline Item 4 Self-perception & $\begin{array}{l}\text { How secure and satisfied the child feels about him/herself as well as appearance reflecting how positively others } \\
\text { value him/her }\end{array}$ \\
\hline Item 5 Autonomy & $\begin{array}{l}\text { The level of the opportunity given to a child to create social and leisure time referring to the child's freedom of } \\
\text { choice, self-sufficiency, and independence }\end{array}$ \\
\hline Item 6 Home life & This attribute examines whether the atmosphere at home is comfortable or not \\
\hline Item 7 Parent relation & This specifies the relationship between the parents and the atmosphere in the child's home \\
\hline Item 8 Peers/social support & $\begin{array}{l}\text { This examines the nature of the child's relationships with other children or peer groups exploring the quality of the } \\
\text { interaction between the child and peers }\end{array}$ \\
\hline Item 9 School environment & How secure and satisfied the child's feeling about the environment the child belonged \\
\hline Item 10 Concentration/learning & How secure and satisfied the ability to concentrate for learning \\
\hline
\end{tabular}


be misfit indicating that the relevant item must have responded in unexpected ways. Rasch model also transform 5-Likert type ordinal scale into invariant interval scale by estimating item difficulty determining more or less challenging in a log odd unit scale (i.e., logit). As item calibrations are presented in order of difficulty from the least to most challenging, those items can be classified into the linear continuum. Thus, one can get a general sense on which domains of KIDSCREEN-10 questionnaire are either least or most challenging to parent and children.

For a traditional approach of concordance analysis between the two reports, IBM-SPSS statistics-version 25 was used to compute descriptive statistics and intra-class correlation coefficients (ICCs) analysis. The pairwise comparisons between two reports were analyzed with ICCs at 95\% confidence intervals (CIs). Due to limited number of sample sizes, 95\% CIs for the ICCs were estimated by bootstrapping method with 1,000 iterations. The degree of correlations was interpreted as: 0-0.19 very weak, 0.20-0.39 weak, 0.40-0.59 moderate, 0.60-0.79 strong and $0.80-1.0$ very strong relationships [18]. For graphical demonstration of concordance in Rasch logit scales between parent proxy and child self-reports, a scatterplot with Rasch logit scales was used.

\section{RESULTS}

As an initial means of item analysis, goodness of fit statistics was examined with the criterion of misfit to determine the dimensionality of two reports of the KIDSCREEN-10-10 questionnaire. Table 2 presents item difficulty calibrations in descending order, Infit/outfit statistics and Z score standardized for two 10 items of the KIDSCREEN-10 questionnaires. All items, except four items of parent proxy report (i.e., psychosocial well-being, mood/emotion, self-conception and parent relation) and three items of child's self-report (i.e., psychosocial well-being, autonomy and home life) exhibit acceptable or slightly out of range in fit statistics. Figure 1 represents the relationship between parent/child's HRQOL status and item difficulty measures in logits. Item difficulty order is similar across the two reports. However, there was big gaps between these two reports in self-perception and mood/emotions items.

Table 3 presents pairwise ICCs between parent proxy and child self-reports ranging from 0.011 to 0.905 . The ICCs of 5 items (i.e, mood/emotions, self-perception, autonomy, home life, and concentration/learning) represent very weak or weak relationships, while other 5 items exhibit moderate to very strong relationships.

A scatterplot of item difficulty estimates in logits between two reports is displayed in Figure 2 to further illustrate how the

Table 2. Fit statistics of the KIDSCREEN questionnaire in descending order of difficulty

\begin{tabular}{|c|c|c|c|c|c|}
\hline Items & Difficulty (logits) & Infit $\mathrm{MnSq}^{\mathrm{a}}$ & ZSTD & Outfit MnSq & ZSTD \\
\hline Concentration/learning (PARENT 10) & 1.07 & 0.51 & -1.03 & 0.75 & -0.35 \\
\hline Autonomy (PARENT 5) & 0.77 & 0.63 & -0.65 & 1.05 & 0.28 \\
\hline Autonomy (CHILD 5) & 0.61 & 2.25 & 1.95 & 1.79 & 1.34 \\
\hline Home life (PARENT 6) & 0.61 & 0.65 & -0.59 & 0.64 & -0.56 \\
\hline Home life (CHILD 6) & 0.44 & 2.20 & 1.88 & 1.48 & 0.91 \\
\hline Concentration/learning (CHILD 10) & 0.44 & 1.33 & 0.74 & 1.25 & 0.60 \\
\hline Self-perception (PARENT 4) & 0.26 & 3.76 & 3.30 & 5.22 & 3.87 \\
\hline Peers/social support (PARENT 8) & 0.26 & 0.58 & -0.75 & 0.42 & -1.06 \\
\hline School environment (PARENT 9) & 0.26 & 0.28 & -1.75 & 0.29 & -1.46 \\
\hline Physical well-being (CHILD 1) & 0.06 & 0.13 & -2.50 & 0.17 & -1.87 \\
\hline Psychological well-being (CHILD 2) & 0.06 & 0.27 & -1.78 & 0.26 & -1.50 \\
\hline Parent relation (CHILD 7) & 0.06 & 0.81 & -0.19 & 0.84 & -0.05 \\
\hline Parent relation (PARENT 7) & 0.06 & 0.38 & -1.34 & 0.38 & -1.10 \\
\hline Peers/social support (CHILD 8) & -0.40 & 0.80 & -0.14 & 0.54 & -0.51 \\
\hline School environment (CHILD 9) & -0.40 & 0.85 & -0.04 & 0.68 & -0.25 \\
\hline Psychological well-being (PARENT 2) & -0.40 & 0.24 & -1.63 & 0.27 & -1.19 \\
\hline Physical well-being (PARENT 1) & -0.70 & 0.46 & -0.79 & 0.40 & -0.67 \\
\hline Mood/emotions (PARENT 3) & -0.70 & 3.01 & 2.21 & 5.93 & 3.22 \\
\hline Mood/emotions (CHILD 3) & -2.35 & 0.45 & -0.33 & 0.18 & -0.45 \\
\hline Self-perception (CHILD 4) & -3.63 & \multicolumn{4}{|c|}{ Minimum measure ${ }^{b}$} \\
\hline
\end{tabular}

MnSq, mean squares; ZSTD, Z score standardized; PARENT, parent proxy report item; CHILD, child self-report item. ${ }^{\text {a }}$ Standardized residuals. ${ }^{b}$ Fit statistics/ZSTDs are too small to estimate. 


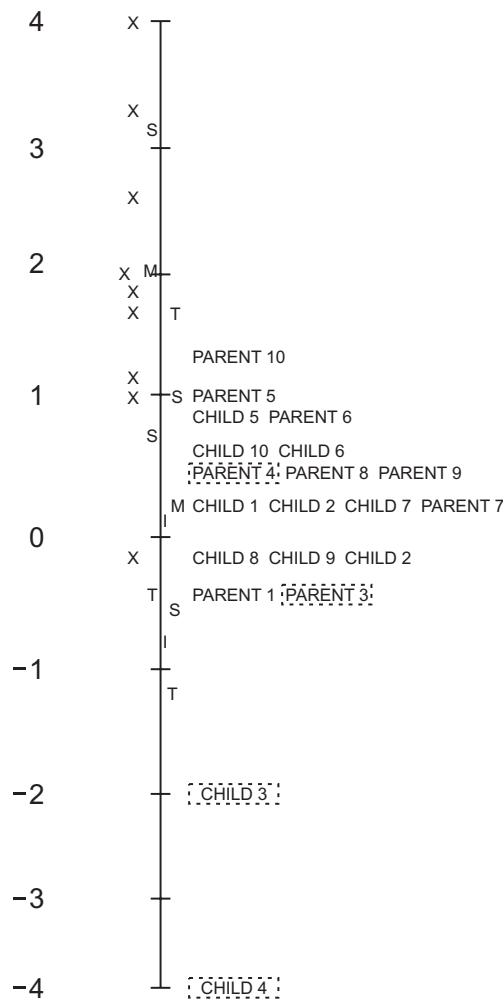

Figure 1. Person-item match for items of parent proxy and child selfreports of the KIDSCREEN-10 questionnaire. The graph represents the relationship among the level of health-related quality of life score and item difficulty calibrations in logits. PARENT, parent proxy report item; CHILD, child self-report item.

two reports are differently responded to parents and children. All items of the two reports, except self-perception item, demonstrate similar item difficulties (i.e. presented with filled dots) within $95 \%$ confidence intervals presented with empty dots. The self-perception item is now flagged as potential disagreement between the two reports. The item is about three logits more difficult when rated by parents.

\section{DISCUSSION}

Overall, the parent proxy and child self-reports of the KIDSCREEN-10 questionnaire showed acceptable some concordances and disparities. As determined by Rasch item-level analysis, high and low fit statistics were encountered in four items of parent proxy and three items of child self-reports. Person-item match across two reports was similar except for two items (i.e., self-perception and mood/emotion). Furthermore, concordances between two reports were determined by ICCs and scatterplot analysis of item difficulty calibrations. The ICCs between two reports on 5 items were of very weak
Table 3. Intra-class correlations (ICC) between scores of parent proxy and child self-reports

\begin{tabular}{lc}
\hline Parent proxy versus child self-report & ICC \\
\hline Physical well-being (Item 1) & $0.743(0.209-0.936)$ \\
Psychological well-being (Item 2) & $0.868(0.523-0.969)$ \\
Mood/emotions (Item 3) & $0.122(-0.553-0.700)$ \\
Self-perception (Item 4) & $0.011(-0.632-0.632)$ \\
Autonomy (Item 5) & $-0.022(-0.645-0.619)$ \\
Home life (Item 6) & $0.161(-0.524-0.720)$ \\
Parent relation (Item 7) & $0.847(0.464-0.963)$ \\
Peers/social support (Item 8) & $0.905(0.639-0.978)$ \\
School environment (Item 9) & $0.695(0.112-0.922)$ \\
Concentration/learning (Item 10) & $0.370(-0.342-0.812)$
\end{tabular}

Values are presented as ICC coefficient (95\% confidence intervals). $p=$ 0.05 .

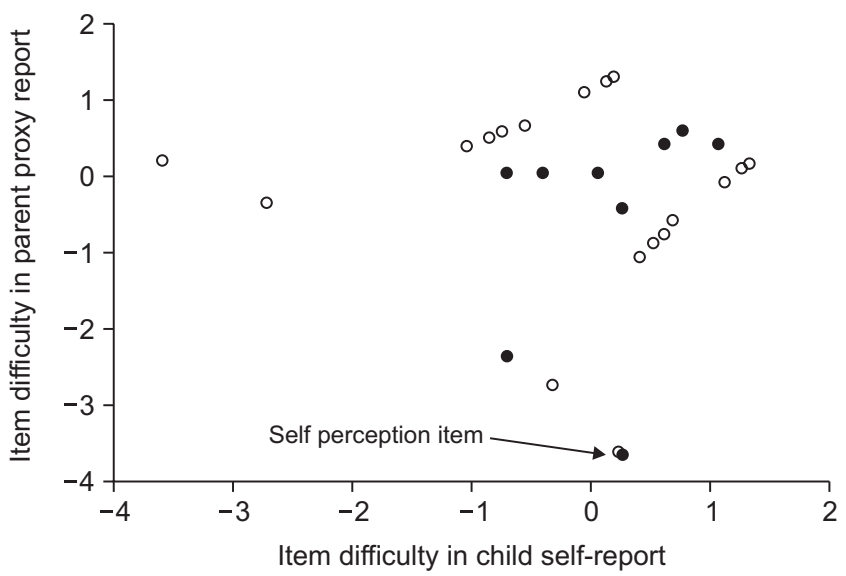

Figure 2. Scatterplot for 10 items of the KIDSCREEN-10 questionnaire across parent proxy and child self-reports at the time of completion of the school-based wellness program. Two lines formed with empty dots represent the upper and lower $95 \%$ confidence intervals. The self-perception item demonstrates different item difficulty between two reports.

to weak relationships, while other 5 items were of moderate to very strong relationships. The scatterplot with Rasch logit scales provided some support for acceptable agreement between two reports, while self-perception item was outside of 95\% confidence intervals.

The present study explored the concordances between parent proxy and child self-reports in individual items of the KIDSCREEN-10 questionnaire. The ICC coefficient and item-level Rasch analysis provided the good overall concordance with some exceptions. The ICCs between parent proxy and child self-reports showed very low concordance on mood/emotions, self-perception, autonomy, home life items and concentration item, while rest of 5 items showed moderate to high concordances. Additionally, Rasch item-level analysis showed similar item difficulty hierarchical structures, however there were 
big gaps between two reports on self-perception and mood/ emotions items in item difficulty calibrations. There were 3.89 logit difference on self-perception and 3.05 logit difference on mood/emotions item. That is, parent proxy report had a tendency to be more challenged than child self-report on those two items. Surprisingly, the two items were also identified as potential problematic items based on fit statistics, scatterplot and person-item match analyses.

These two items are of concern. First, why is there a disparity across two reports on those two items? If such a disparity between two reports on two items exist, which report would be more valid? This disparity may be result of differing perspectives on the items. Parents are likely to be carefully specific interpretation on self-perception and mood/emotions items, while children are likely to be intuitively interpret them although perception toward those items are challenging to elementary school children. Those items may be misinterpreted by most children. Secondly, one of the most critical perspectives of KIDSCREEN-10 questionnaire is the concordance issue as to whether parallel two reports would be able to measure same construct or not $[19,20]$. Rasch fit statistics were examined to determine dimensionality of two reports. Using Bond and Fox's criterion for fit statistics, four items from parent proxy and 3 items from child self-report were misfit. In general, fit statistic provides an indication of observed variance over expected variance [21]. High infit suggests that there may be an unusual pattern of responses for 4 items (i.e., autonomy, home life, self-perception and mood/emotions) when attempting to assess parents and children at HRQOL levels similar to the items. In this study, high outfit of the 7 items indicates that there are unusual response patterns for the items when attempting to assess parents and children at HRQOL levels distance from the items. For instance, since mood/emotions item is among the easiest of the items, this would suggest that parent/children with high HRQOL may show high ratings (i.e., selecting always feeling) on the items. However, lower ratings may be selected by most parent/children with being high fit statistics. In contrast to items with high fit statistics (i.e., overfitting), if items with low fit statistics shows too little variation (i.e., underfitting) such as self-conception and mood/emotions of child self-report, this would indicate unexpectedly perfect responses. This measurement problem, in general, may be arisen from the limited number of sample size.

Additionally, although several studies confirm evidences of moderate to strong correlations between parent proxy and child self-reports $[11,22,23]$, few authors indicate that the degree of concordance between parent proxy and child self-reports is dependent upon the domain being measured [24]. That is, parent proxy report tended to be more serious than child self-report for domains concerning functional limitations and to be less serious for domains concerned with emotional and social well-being [24]. Despite those disparities between two reports, information provided by parent is still recommended to be used in evaluating HRQOL of children [11,24]. The present study results showed more disparities than the previous studies' findings $[11,22,23]$. Several reasons can be postulated for having more disparities in this study. First, cross-culturally adapted versions of the KIDSCREEN-10 questionnaires used for the present study are validated for individuals with various impairments. Two focus groups in this study consisted of healthy persons without any known impairment. Since there were no such thing as specific impairments other than general poor postures or mild discomforts in children's daily life, two reports had no known common issue when parents and children were asked to think about relevant items within the KIDSCREEN-10 questionnaire. Future versions of it may require further validations reflecting norm groups. Second, this exploratory pilot study is initiated to examine whether elementary school-based wellness programs are feasible to be extended in the whole school districts. Given limited current resources of a local school participated for this study, it was partially feasible to examine the concordance and the item level analyses on both HRQOL reports.

The present study used data from a larger project to develop a school-based wellness program with screening services as well as home exercises in necessary. Elementary school-based focus group with risk factors for potential musculoskeletal impairments was recruited from an urban area in Daejeon, Republic of Korea. While the project was originally designed to include many elementary schools in the area, only one school was actually participated in the project. This limited number of participating schools may cause the statistical bias in concordances between two reports. Limitations of the present study include high/low fit statistics and very weak and weak relationships between two reports probably due to limited sample size and non-impairment focus group recruitment. Thus, future study should examine the disparity between two reports with proper focus groups selection reflecting various impairments 
and acceptable sample size.

\section{CONCLUSIONS}

This study explored the differences between parent proxy and child self-reports of the KIDSCREEN-10 questionnaire using Item level analyses as well as ICCs coefficients. The study provides some evidences of a rating disparity between two reports. Four items of parent proxy report (i.e., psychological well-being, mood/emotions, self-perception, parent relation items) were found to be problematic, while three items from child self-report (i.e., psychological well-being, autonomy and home life items) were noisy based on fit statistics. There were weak to very strong relationships of ICCs between the two reports ranging from 0.011 to 0.905 . Scatterplot analysis between two reports showed a major disparity on self-perception item at 95\% confidence intervals. Future studies should consider indepth investigations with acceptable focus groups with various impairments and suitable sample sizes.

\section{ACKNOWLEDGEMENTS}

This research is based on the support of 2021 Woosong University Academic Research Funding.

\section{CONFLICTS OF INTEREST}

No potential conflict of interest relevant to this article was reported.

\section{REFERENCES}

1. Tercedor P, Villa-González E, Ávila-García M, Díaz-Piedra C, Martínez-Baena A, Soriano-Maldonado A, et al. A school-based physical activity promotion intervention in children: rationale and study protocol for the PREVIENE Project. BMC Public Health 2017;17(1):748.

2. Kugel J, Hemberger C, Krpalek D, Javaherian-Dysinger H. Occupational therapy wellness program: youth and parent perspectives. Am J Occup Ther 2016;70(5):7005180010p1-8.

3. Clark GF, Watling R, Parham LD, Schaaf R. Occupational therapy interventions for children and youth with challenges in sensory integration and sensory processing: a schoolbased practice case example. Am J Occup Ther 2019;73(3): 7303390010p1-8.

4. Solans M, Pane S, Estrada MD, Serra-Sutton V, Berra S, Herdman M, et al. Health-related quality of life measurement in children and adolescents: a systematic review of generic and disease-specific instruments. Value Health 2008;11(4):74264.

5. Goldthorpe J, Epton T, Keyworth C, Calam R, Armitage C. Who is responsible for keeping children healthy? A qualitative exploration of the views of children aged 8-10 years old. BMJ Open 2019;9(5):e025245.

6. Ravens-Sieberer U; Kidscreen Group Europe. The Kidscreen questionnaires: quality of life questionnaires for children and adolescents: handbook. Lengerich: Pabst Science; 2006.

7. Davis E, Nicolas C, Waters E, Cook K, Gibbs L, Gosch A, et al. Parent-proxy and child self-reported health-related quality of life: using qualitative methods to explain the discordance. Qual Life Res 2007;16(5):863-71.

8. Theunissen NC, Vogels TG, Koopman HM, Verrips GH, Zwinderman KA, Verloove-Vanhorick SP, et al. The proxy problem: child report versus parent report in health-related quality of life research. Qual Life Res 1998;7(5):387-97.

9. Bastiaansen D, Koot HM, Ferdinand RF. Determinants of quality of life in children with psychiatric disorders. Qual Life Res 2005;14(6):1599-612.

10. Brunner HI, Klein-Gitelman MS, Miller MJ, Trombley M, Baldwin N, Kress A, et al. Health of children with chronic arthritis: relationship of different measures and the quality of parent proxy reporting. Arthritis Rheum 2004;51(5):763-73.

11. Eiser C, Morse R. Can parents rate their child's health-related quality of life? Results of a systematic review. Qual Life Res 2001;10(4):347-57.

12. Liang MH, Lew RA, Stucki G, Fortin PR, Daltroy L. Measuring clinically important changes with patient-oriented questionnaires. Med Care 2002;40(4 Suppl):II45-51.

13. McHorney CA. Health status assessment methods for adults: past accomplishments and future challenges. Annu Rev Public Health 1999;20:309-35.

14. Gamper EM, Groenvold M, Petersen MA, Young T, Costantini A, Aaronson N, et al.; EORTC Quality of Life Group. The EORTC emotional functioning computerized adaptive test: phases I-III of a cross-cultural item bank development. Psychooncology 2014;23(4):397-403.

15. Müller M, Haenni Hoti A. Item analysis of the KIDSCREEN-10 using Rasch modelling. Health Qual Life Outcomes 2020; 
18(1):342.

16. Hong SD, Yang JW, Jang WS, Byun H, Lee MS, Kim HS, et al. The KIDSCREEN-52 quality of life measure for children and adolescents (KIDSCREEN-52-HRQOL): reliability and validity of the Korean version. J Korean Med Sci 2007;22(3):446-52.

17. Bond TG, Fox CM. Applying the Rasch model: fundamental measurement in the human sciences. New York: Routledge; 2015;26-7.

18. Evans JD. Straightforward statistics for the behavioral sciences. Pacific Grove: Brooks/Cole; 1996;43-56.

19. Ravens-Sieberer U, Erhart M, Rajmil L, Herdman M, Auquier P, Bruil J, et al.; European KIDSCREEN Group. Reliability, construct and criterion validity of the KIDSCREEN-10 score: a short measure for children and adolescents' well-being and health-related quality of life. Qual Life Res 2010;19(10):1487500.

20. Ravens-Sieberer U, Auquier P, Erhart M, Gosch A, Rajmil L, Bruil J, et al.; European KIDSCREEN Group. The KID-
SCREEN-27 quality of life measure for children and adolescents: psychometric results from a cross-cultural survey in 13 European countries. Qual Life Res 2007;16(8):1347-56.

21. Linacre JM. Teaching Rasch measurement. Rasch Meas Trans 2017;31(2):1630-1.

22. Stevanovic D, Tadic I, Novakovic T, Kisic-Tepavcevic D, RavensSieberer U. Evaluating the Serbian version of the KIDSCREEN quality-of-life questionnaires: reliability, validity, and agreement between children's and parents' ratings. Qual Life Res 2013;22(7):1729-37.

23. Erhart M, Ottova V, Gaspar T, Jericek H, Schnohr C, Alikasifoglu M, et al.; HBSC Positive Health Focus Group. Measuring mental health and well-being of school-children in 15 European countries using the KIDSCREEN-10 Index. Int J Public Health 2009;54 Suppl 2:160-6.

24. Jokovic A, Locker D, Guyatt G. How well do parents know their children? Implications for proxy reporting of child healthrelated quality of life. Qual Life Res 2004;13(7):1297-307. 